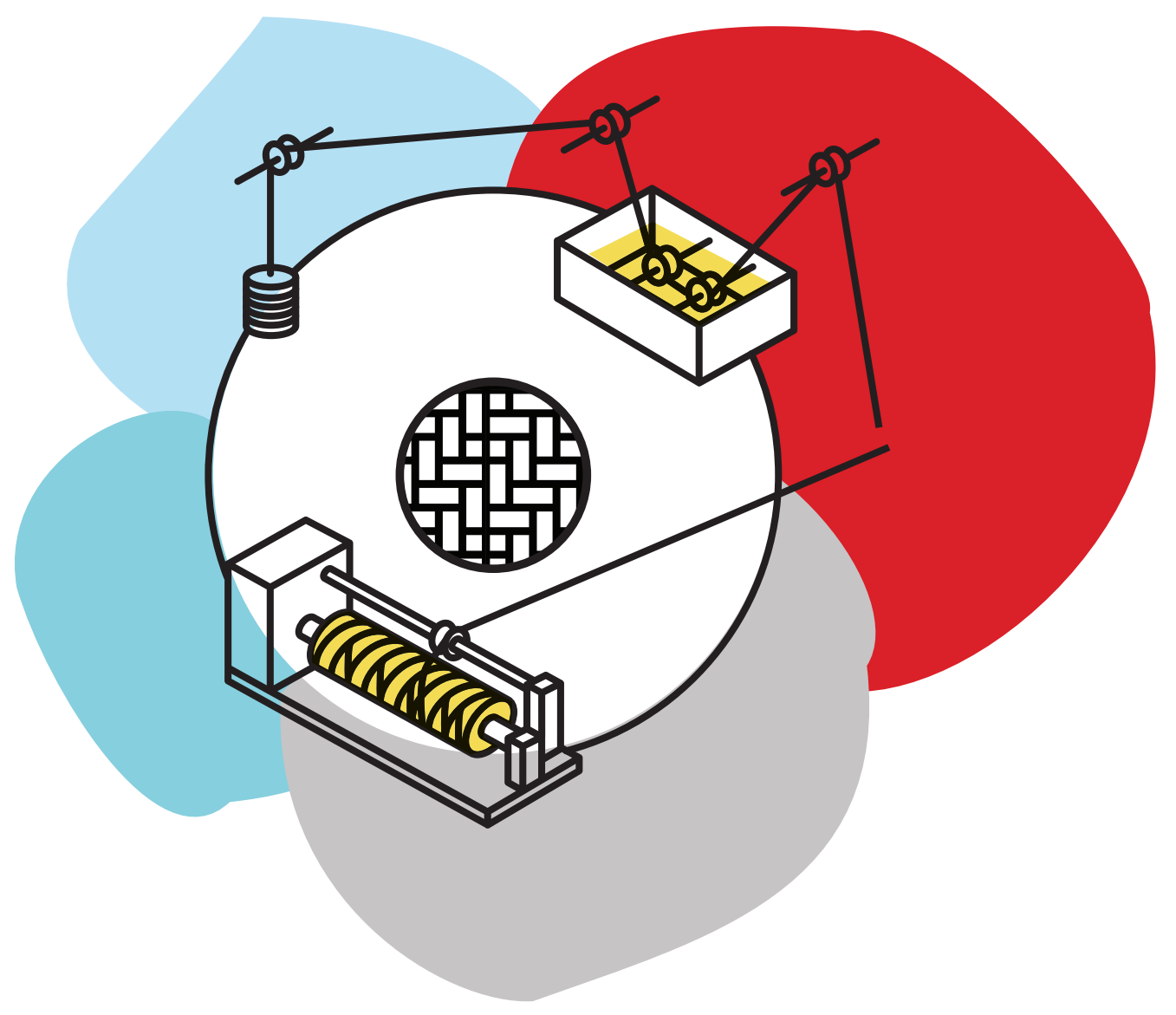

\title{
EL PLÁSTICO REFORZADO CON FIBRA DE VIDRIO: UN MATERIAL VERSÁTIL OLVIDADO EN URUGUAY
}

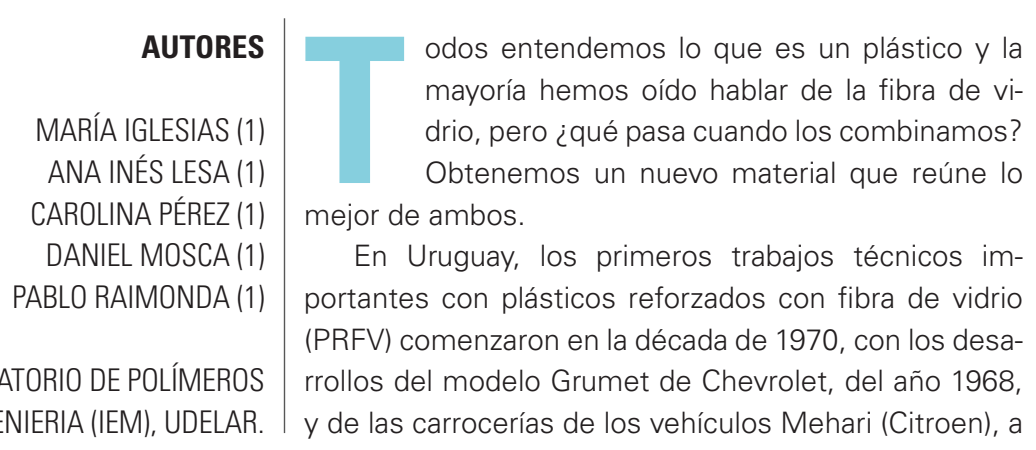


cargo de la firma DASUR S.A. En la misma época, en los talleres de CUTCSA se desarrolló el ómnibus Banda Oriental 70, con frente y paneles traseros y asientos enterizos de este material, construidos sobre un chasis Leyland. Por entonces también recibió fuerte impulso en Uruguay el desarrollo de la industria naval liviana.

Atendiendo a esta realidad, desde la Universidad de la República (UdelaR), en 2002 se creó en Facultad de Ingeniería una asignatura dedicada a capacitar en el tema a los futuros ingenieros.

Las circunstancias de mercado hicieron que estas empresas pioneras dejaran de producir estos componentes, lo cual se reflejó también a nivel académico en la cantidad de alumnos inscriptos en esta unidad curricular.

En los años siguientes, este material ha avanzado a un ritmo exponencial, sin embargo, en Uruguay los conocimientos han mantenido la impronta de la década de 1980.

En 2017, en el contexto del Instituto de Ensayo de Materiales de la Facultad de Ingeniería se creó un equipo de trabajo con el desafio de acompañar el estado del arte en este campo.

\section{¿Qué es un plástico reforzado con fibra de vidrio?}

Se entiende por material compuesto aquel que está formado por dos o más componentes, de manera que las propiedades del material final sean superiores a las de los componentes por separado (Miravete, 2015).

Los desarrollos en el campo tecnológico dependen de los avances en el campo de los materiales. No es necesario ser experto para notar que, por ejemplo, las turbinas más avanzadas o el diseño de los aviones no hubieran llegado a su potencial actual sin los materiales adecuados. Desde este punto de vista, los materiales compuestos representan un paso gigante en el constante camino de optimización de los recursos a emplear, y forman cada vez más parte de nuestra vida cotidiana. Al subir a un ómnibus, por ejemplo, estamos rodeados de componentes fabricados por materiales compuestos, mientras que en un avión comercial de última generación más del 50 \% de la estructura está formada por estos materiales.

La idea de los materiales compuestos no es reciente. En la naturaleza existe una gran cantidad de materiales de este tipo: los huesos, el bambú, las fibras naturales y la madera son algunos de ellos. Los huesos son un compuesto de proteína (colágeno) y mineral (apati- ta). El bambú es celulosa reforzada con sílice, combinación que hace del bambú un material duro, con alta resistencia al impacto. En la estructura de la madera, la celda de celulosa y la fibra están unidas con lignina, una sustancia polimérica natural. Las celdas de la madera son estructuras compuestas naturales en sí mismas.

En el caso del plástico reforzado con fibra de vidrio (PRFV), se trata del uso de las fibras de vidrio como refuerzo de una matriz polimérica que actúa como aglomerante. Esta fibra provee al compuesto resistencia mecánica, estabilidad dimensional y resistencia al calor, mientras que la matriz aporta resistencia química, dieléctrica, y resistencia a la intemperie.

Lo que se busca con los materiales compuestos o composites es obtener un producto con propiedades que generalmente no se encuentran en un solo material, logrando con ello un efecto sinérgico entre las mejores propiedades de cada uno, por ejemplo, la alta resistencia mecánica, la dureza y el bajo peso simultáneos.

Su utilización es frecuente en la industria aeroespacial para la disminución del peso, como alternativa a los compuestos estructurales usuales como el acero, o en estructuras tales como los puentes, las cañerías de desagüe y las vigas, en sustitución del hormigón o la madera. También se encuentran en chalecos antibala, equipamientos para deportes -la garrocha en atletismo-, chasis de la fórmula 1, embarcaciones pesqueras, o industrias del transporte.

Los composites pueden ser clasificados de distintas formas: según el tipo de matriz utilizada (polimérica, cerámica o metálica), o según la morfología y configuración del refuerzo aplicado, que puede tener una estructura de tipo partículas, de fibras o láminas. A su vez, también es posible abrir una segunda clasificación para el caso particular de las fibras de acuerdo a cómo se presentan: continuas o discontinuas, unidimensionales, bidimensionales o tridimensionales.

A diferencia de la producción de piezas con otros materiales, cuando se utilizan composites las propiedades de las piezas no solo dependen de la composición del compuesto, sino de otros factores, como el método de fabricación, el tipo y forma de la fibra, o su orientación en la matriz. Por ejemplo, cuando se utiliza fibra de vidrio (PRFV) en forma de fibras continuas éstas presentan excelentes propiedades para ser utilizadas en estructuras. Sin embargo, si la misma fibra es aplicada en forma discontinua y al azar, el producto pierde propiedades estructurales (Miravete, 2015). 
Otro ejemplo de cómo pueden verse afectadas las propiedades es en el moldeado. Cuando realizamos un laminado manual éste puede contener un máximo de 30 $\%$ de fibras, mientras que si lo hacemos por el proceso de confección dentro de un molde cerrado y con un saco de vacío se puede llegar hasta un 50 \% de fibra (RTM-light; Miravete, 2015).

Para poder diseñar estructuras de PRFV de forma eficiente es necesario seguir, entonces, los siguientes pasos:

- Conocer los requisitos que debe cumplir la estructura (resistencia a la tracción, deflexión máxima, geometría básica, etcétera).

- Definir el tipo de composite (PRFV, polywood, material compuesto con fibra de carbono, etcétera) y su método de conformación.

- Calcular la estructura.

- Realizar ensayos de aptitud.

A modo de ejemplo, pensemos en el diseño de las patas de un banco. Para lograr el resultado esperado es necesario conocer, o definir entre las distintas variables a priori, el peso que tiene que soportar el banco, para que sea bien distribuido entre las patas, o la cantidad de soportes que se deben colocar y su sección para que cumplan estas condiciones prefijadas. Para los demás componentes se deberán repetir los mismos pasos. Con esta información será posible construir un prototipo que será verificado mediante ensayos adecuados para confirmar el cumplimiento de las condiciones definidas en el diseño.

De los distintos tipos de combinaciones de las materias primas que se pueden utilizar para confeccionar un material compuesto, el plástico reforzado, que es objeto de este artículo, se caracteriza por la utilización de polímeros termorrígidos con inclusión de fibras de vidrío.

A continuación aludiremos a cada uno de los componentes del composite: fibras y matriz.

En cuanto a las fibras, las características más importantes son su resistencia a la tracción y su elevado módulo específico, propiedades que se manifiestan en su dirección longitudinal. Estos refuerzos, para ser seleccionados como tales, deben ser resistentes, rígidos, ligeros y con una temperatura de fusión alta. La influencia de las fibras sobre las propiedades del composite es definitiva en cuanto a los requerimientos a lograr.
Las propiedades del composite varían en función del contenido de fibra en la matriz, pero este incremento no es lineal, sino que hay un valor máximo que luego tiende a decaer al sobrepasar determinadas concentraciones de fibra. Cabe recordar que frente a cualquier porcentaje de fibra utilizada siempre se debe asegurar que la totalidad de su superficie quede recubierta con el material de la matriz. Estudios realizados indican que este límite máximo de concentración de fibra suele establecerse en el orden del 80 \% (Olivares, et al., 2003).

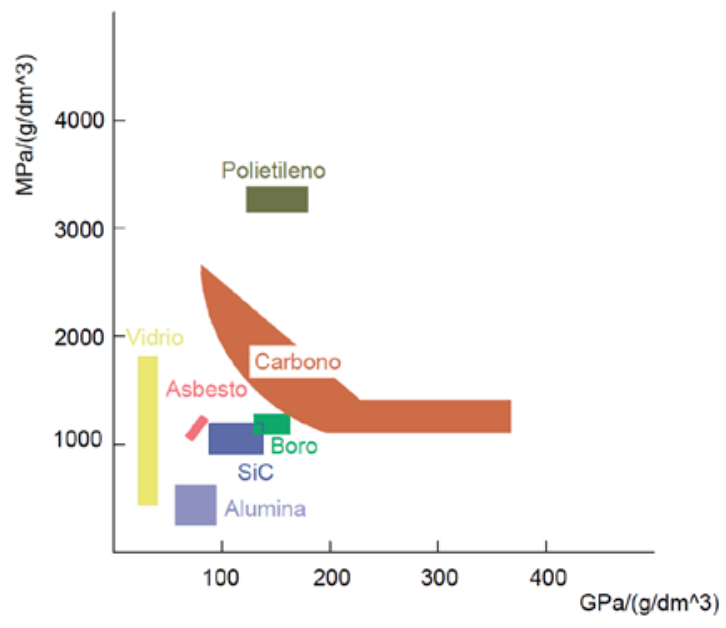

Figura 1. Comparación de las características de las dierentes fibras.

Un aspecto a tener en cuenta y que afecta las propiedades mecánicas del composite es la orientación u ordenamiento de la fibra dentro de la matriz. Esta cualidad que presenta el material compuesto de variar los valores de sus propiedades respecto a la orientación del material de refuerzo es lo que en ciencia de los materiales se denomina anisotropía.

El otro elemento integrante del composite es la matriz o aglutinante, cuya función es mantener las fibras unidas y transferir las cargas exteriores a los refuerzos. Las fibras reforzantes no pueden soportar por sí solas los esfuerzos del tipo transversales ni los de compresión, algo que sí lo puede hacer la estructura de la matriz. La rigidez y resistencia transversal se deben exclusivamente a este aglutinante.

Otra de las funciones de la matriz es proteger a las fibras de las condiciones medioambientales, para evitar la degradación y, por consiguiente, la pérdida de propiedades de los materiales compuestos. Por otra parte, la 
matriz juega un papel crucial en lo referente a la conductividad térmica y eléctrica del material.

En Uruguay, el PRFV se utiliza principalmente para la fabricación de recipientes, tanto para la industria química como para la alimenticia. También se aplica en otros rubros, para la producción de tableros eléctricos y reparación de embarcaciones navales. Con la instalación del primer parque eólico de UTE en el año 2008, comenzaron a verse en el país, aunque importadas, las primeras grandes estructuras realizadas con estos materiales. Por otra parte, bajo la tutoría de los autores de este artículo, en el año 2015 alumnos de Facultad de Ingeniería demostraron en el marco de un trabajo de grado que la construcción de señales de tránsito con estos materiales tiene un costo $33 \%$ menor a las construidas tradicionalmente en chapa tratada. De todos modos, la industria nacional de los PRFV sigue sin despegar, salvo contadas excepciones. En la mayoría de los casos se trata de pequeños talleres totalmente artesanales. La calidad del producto obtenido en estas microempresas no es la mejor; su principal carencia es la no realización de cálculos estructurales de los productos fabricados.

Para el cálculo de este tipo de estructuras se debe tener en cuenta la gran variedad de formas de los reforzantes. Es necesario prestar especial atención a este detalle, ya que la acción reforzante depende de la relación largo/ancho de la fibra (K.K. Charla). Incluso partículas con relaciones de aspecto iguales pueden tener formas tan diferentes como esferas, elipses, cubos, o irregulares, como lo describen Kumar et al. (2009). Por tal motivo, algunas variables clave para una correcta determinación de las propiedades de los materiales compuestos son la fracción volumétrica y la forma del reforzante, incluidas en varios modelos teóricos (Halpin y Kardos, 1976; Hashin y Shtrikman, 1963; Mura, 1987; Tsai-Wu, et al., 1971; Lewis y Nielsen, 1970).

Para el cálculo de estructuras de geometrías complicadas existen tres métodos distintos: el método de elementos finitos (FEM), el de volúmenes finitos (FVM) y el de diferencias finitas (FDM). La diferencia principal entre los tres readica en cómo toman en los cálculos la variable independiente. El más utilizado en materiales compuestos es el FEM. Numerosos trabajos utilizan el método de los elementos finitos para estimar las propiedades de los materiales compuestos (García de la Figal y Frías, 2003; Oprisan, et al., 2016, Bačinskas, et al., 2017; Ševčík, et al., 2015; Correia, et al., 2015; Yang, X., et al., 2015; Nunes, et al., 2015; Santiuste, et al., 2010).

En los materiales compuestos las propiedades finales son dependientes del método y del proceso de producción. Para alcanzar mejores resultados es necesario parametrizar nuestros modelos a la realidad uruguaya, que en la mayoría de los casos es muy distinta a la empleada en la construcción de las bases de datos internacionales, como la del Sandia National Laboratories, de EEUU, especializados en el ensayo de palas de aerogeneradores, o la base de Datos Matweb, en forma más general. Luego de la simulación se deben interpretar los resultados comparándolos mediante ensayos con los requisitos definidos anteriormente.

Por último, es necesario validar nuestro diseño mediante la realización de un prototipo y los ensayos correspondientes de similitud, para así poder determinar si en la realidad se cumplen las condiciones que en el diseño se dispusieron como necesarias.

Para generar una masa crítica que pueda enfrentar estos nuevos desafíos, el Instituto de Ensayo de Materiales de la Facultad de Ingeniería de UdelaR ha comenzado hace algún tiempo a implementar estas herramientas en la formación de los nuevos ingenieros. Entre los cambios más significativos se encuentra precisamente la capacitación en la modelación por FEM. A tales efectos ya se encuentra trabajando un grupo con el objetivo de especializarse en estos temas.

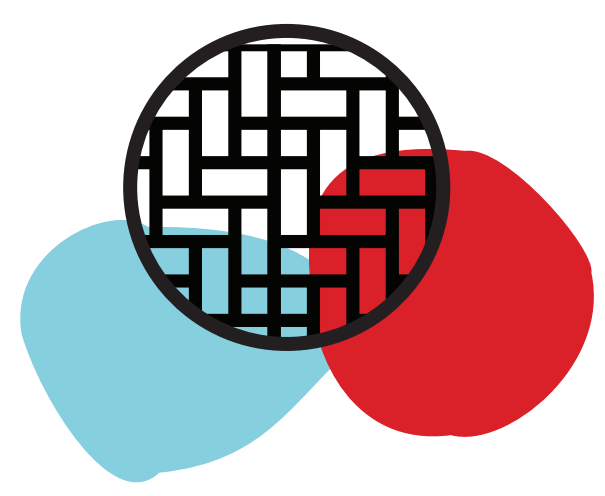




\section{Referencias}

Bačinskas, D., Rimkus, A., Rumšys, D., Meškènas, A., Bielinis, S., Sokolov, A. y Merkevičius, T., 2017. Structural analysis of GFRP truss bridge model. En: Procedia Engineering, 172, pp.68-74.

Correia, J.R., Bai yYu, Keller, T., 2015. A review of the fire behaviour of pultruded GFRP structural profiles for civil engineering applications. En: Composite Structures, 127, pp.267-287.

Chawla, K. K., 2013. Composite materials. New York: Springer. ISBN 978-0-387-74365-3

García de la Figal, J. y Frías, O., 2003. Cálculo de patana especializada construida de PRFV. En: Ingeniería Mecánica, 6 (2), pp.65-72

Gol'denblat, I.I. y Kopnov, V.A., 1971. General theory of criteria of strength for isotropic and anisotropic materials. En: Strength of Materials, 3(2), pp.184-188.

Halpin, J.C. y Kardos, J.L., 1976. The Halpin-tsai equations: a review. En: Polymer Engineering And Science, 16(5), pp.344-352.

Hashin, Z. y Shtrikman, S., 1963. A variational approach to the theory of the elastic behaviour of multiphase materials. En: Journal of the Mechanics and Physics of Solids, 11(2), pp.127-140.

Kumar, A.P., Depan, D., Tomer, N.S. y Singh, R.P., 2009. Nanoscale particles for polymer degradation and stabilization-Trends and future perspectives. En: Progress in Polymer Science, 34, pp.479-515.

Lewis. T.B. y Nielsen. L.E., 1970. Dynamic mechanical properties of particulate-filled composites. En: Journal of Applied Polymer Science, 14(6), pp.1449-1471.

Miravete Antonio, 2015. Materiales compuestos. Barcelona: Reverté. ISBN 978-84-921349-7-7.

Mura, T., 1987. Micromechanics of defects in solids. 2 ed. Nueva York: Springer.
Nunes, F., Correia, M., Correia, J.R., Silvestre, N. y Moreira, A., 2013. Experimental and numerical study on the structural behavior of eccentrically loaded GFRP columns. En: Thin-Walled Structures, 72, pp.175-187

Olivares, S. M., Galán, M. y Fernández, J. R., 2003. Composites: characterístics and applications in building construction. En: Informes de la Construcción, 54(484).

Oprisan, G., Ţaranu, N., Mihai, P., Sorina, I., Maxineasa, S.G. y Lupasteanu, V., 2016. Structural response of pultruded GFRP profiles subjected to bending. En: Buletinul Institutului Politehnic DIN IAŞI, 62(66), Numărul 3, 2016.

Santiuste, C., Soldani, X., Henar, M.M. y Soldani, X., 2010. Machining FEM model of long fiber composites for aeronautical components. En: Composite Structures, 92 (3), pp.691-698.

Ševčík, M., Huta, P., Vassilopoulos, A.P. y Shahverdi, M., 2015. Analytical model of asymmetrical MixedMode Bending test of adhesively bonded GFRP joint. En: Frattura ed Integrità Strutturale, 34, pp.216-225. DOI: 10.3221/IGF-ESIS.34.23

Tsai, S. y Wu, E., 1972. A general theory of strength for anisotropiv materials, laboratorio de materiales. En: J. Composite Materials, 5, pp.58-80.

Yang, X., Bai, Y. y Ding, F., 2015. Structural performance of a large-scale space frame assembled using pultruded GFRP composites. En: Composite Structures, 133, pp.986-996 